\title{
Commensal Searches for Extraterrestrial Intelligence with Arecibo Observatory and the Green Bank Telescope
}

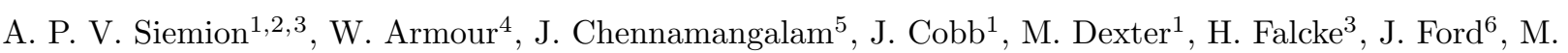

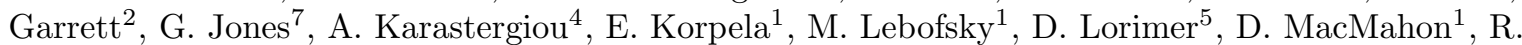 \\ Maddalena ${ }^{6}$, M. McLaughlin 5 , and D. Werthimes ${ }^{10}$ \\ ${ }^{1}$ University of California, Berkeley, Berkeley, California, United States \\ ${ }^{2}$ ASTRON, Dwingeloo, Netherlands \\ ${ }^{3}$ Radboud University, Nijmegen, Netherlands \\ ${ }^{4}$ Oxford University, Oxford, United Kingdom \\ ${ }^{5}$ West Virginia University, Morgantown, West Virginia, United States \\ ${ }^{6}$ National Radio Astronomy Observatory, Green Bank, West Virginia, United States \\ ${ }^{7}$ Columbia University, New York, New York, United States
}

\begin{abstract}
Our group is currently developing two new instruments to conduct commensal searches for extraterrestrial intelligence (SETI) at the Green Bank Telescope and Arecibo Observatory. The SETI sky surveys conducted with these two instruments will use all available receivers at these two facilities and target a range of signal types. When complete, these surveys will be the most sensitive and thorough sky surveys for radio emission produced by extraterrestrial technology ever conducted. Here we describe the technical details of our new digital instruments, including the signal acquisition, distribution and science processing components. We also discuss expected science returns and potential expansions to the digital system that could enable wider bandwidths and allow other observers to use the system for their own commensal programs with a minimal investment in new hardware.
\end{abstract}

\section{Introduction}

Millennia after human beings first considered their place in the cosmos, the prevalence or scarcity of life outside the Earth remains one of the most compelling and elusive questions humanity has sought to answer. Today, modern astronomy and astrobiology are at the front lines of this exploration along several fronts, including in-situ measurements on the surfaces and in the atmospheres of other bodies in our own solar system, remote sensing of extrasolar planet atmospheres and direct searches for evidence of advanced extraterrestrial technology (SETI). Though we seek to understand the cosmological distribution of life in all its forms, it is undeniable that very complex extraterrestrial life, notably life that is self-aware and develops technology, is particularly interesting in its potential similarities with our own species. The discovery of intelligent life on another world would be among the most impactful discoveries in the history of science.

Radio astronomy has long played a prominent role in searches for extraterrestrial intelligence (SETI), beginning with the first suggestions by Cocconi and Morrison [1 that narrow-band radio signals near 1420 $\mathrm{MHz}$ might be effective tracers of advanced technology and early experiments along these lines by Frank Drake in 1961 [2, continuing through to more recent investigations searching for a variety of coherent radio signals indicative of technology at a wider range of frequencies, e.g. [3, 4, 5, 6, 7]. The motivation for radio searches for extraterrestrial intelligence has been throughly discussed in the literature 8, 10, 11, 12, 13, 14, but the salient arguments are the following: 1. coherent radio emission is commonly produced by advanced technology (judging by Earth's technological development), 2. electromagnetic radiation can convey information at the maximum velocity currently known to be possible, 3. radio photons are energetically cheap to produce, 4 . certain types of coherent radio emissions are easily distinguished from astrophysical background sources, especially within the so-called "terrestrial microwave window" and, 5. these emissions can transit vast regions of interstellar space relatively unaffected by gas, plasma and dust.

The SETI search space is so broad in many dimensions, including both frequency and sky location, that a proper search requires very large amounts of telescope time. Yet the dedication of significant amounts of observing time on the largest publicly-funded radio telescopes to a single science program, let alone one as speculative as SETI, is simply not possible. More than 30 years ago, SETI astronomers devised a solution that they dubbed "parasitic SETI." By taking advantage of the fact that the amplified sky signal from a radio telescope can be duplicated many times over with very little added noise or loss of sensitivity, SETI astronomers could piggyback on other users observations to conduct vast sky surveys for signs of intelligent life without ever billing a minute of primary-user time. Now more appropriately called commensal observing, this technique has enabled the SERENDIP, SETI@home and AstroPulse projects to use the Arecibo Observatory to conduct the deepest and most comprehensive radio SETI sky survey in history [5].

\section{New Instruments}

In early 2014, our group is deploying two new commensal instruments at Arecibo Observatory and the Green Bank Telescope that will be used to conduct simultaneous searches for extraterrestrial intelligence and fast radio transients, the latter specifically designed to detect more of the recently identified fast radio bursts (FRBs) [15]. This system uses a single set of analog-digital converters and digital signal processing electronics to digitize, coarsely channelize and distribute time domain or full-Stokes integrated data products to CPU/GPU 
processing engines via an Ethernet switch (Figures 1, 2, and 3).

Figures 4 and 5 depict the commensal systems with two processing engines, the SETI SERENDIP 6 (S $\left.\mathrm{S}_{\mathrm{VI}}\right)$ cluster and the ARTEMIS fast radio burst search system. Both the ARTEMIS and SERENDIP engines operate completely independently from one another. The overall system is capable of supporting additional Ethernet subscribers to the coarse channelized data for other experiments.

The $\mathrm{S}_{\mathrm{VI}}$ engine is the latest SETI instrument developed as part of the 30+ year-old Search for ExtraTerrestrial Radio Emissions from Nearby Developed Intelligent Populations (SERENDIP) program [16. In addition to performing sub- $\mathrm{Hz}$ spectroscopy, baseline subtraction and thresholding over the full $2.5 \mathrm{GHz} \mathrm{BW}$, the new instrument features real-time RFI excision capabilities, the ability to buffer raw time-domain data and perform triggered write-to-disk and an architecture that will eventually allow us to implement additional cyclostationary detection algorithms, e.g. autocorrelation [17. The ARTEMIS fast radio transient search system performs realtime dedispersion, thresholding and pulse searching and can optionally trigger captures of raw full-Stokes data if a high confidence event is discovered [18].

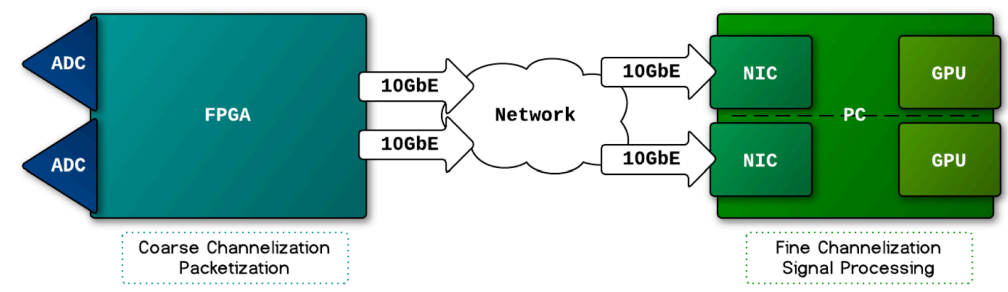

Figure 1: High level system architecture diagram. Field Programmable Gate Array (FPGA)-equipped DSP boards perform signal acquisition and distribution (Figures 2 and distribute data to a bank of commodity CPU/GPU nodes for signal detection and logging. (Figure 3).

For SETI observations at Arecibo, up to eight $448 \mathrm{MHz}$ dual-polarization bands can be channelized down to sub-Hz resolution and searched for emission that could originate with an extraterrestrial technology. For radio transient searches, the same bands are channelized at $\sim 100 \mathrm{kHz}$ resolution, detected and run through a dispersion measure search to identify radio pulses that show the tell-tale signs of alteration due to their transit through a column of free electrons in the interstellar or intergalactic medium. Our Arecibo system (Figure 5) has been specifically designed to accommodate the complete analog output of the Arecibo L-band Feed Array (ALFA) but we will use components of it to process signals from all available receivers. An additional computing node has been incorporated into the system to perform high-level interference rejection and transmit event alerts to other telescopes for followup. At the Green Bank Telescope, a similar system to that described above will process one $2.5 \mathrm{GHz}$ dual polarization band (Figure 4 ).

\section{Sensitivity}

The minimum detectable flux, $F_{i}$, of a narrow band signal in a single polarization is roughly given by 1

$$
F_{i}=\sigma_{\text {thresh }} S_{\mathrm{sys}} \sqrt{\frac{\Delta b}{t}}
$$

Where $\sigma_{\text {thresh }}$ is the signal/noise threshold, $S_{\text {sys }}$ is the system equivalent flux density (SEFD) of the receiving telescope, $\Delta b$ is the spectral channel bandwidth and $t$ the integration time.

Figure 6 shows the minimum detectable equivalent isotropically radiated power (EIRP) using available receivers at Arecibo Observatory and on the Green Bank Telescope, for a transmitter at 5 pc. We have assumed a $15 \sigma$ detection threshold and a $300 \mathrm{~s}$ incoherent integration on a continuous-wave sinusoid with $0.8 \mathrm{~Hz}$ channel width. We have used reference system temperature and gain values, neglected galactic synchrotron background and for frequencies above $15 \mathrm{GHz}$ we assume a $50 \%$ weather quantile at the GB site [19]. For reference, a characteristic EIRP for high-power terrestrial surveillance radars is $\sim 10^{17} \mathrm{erg} \mathrm{s}^{-1}$, and the EIRP of the Arecibo Planetary Radar is $\sim 2 \times 10^{20} \mathrm{erg} \mathrm{sec}^{-1}$.

\footnotetext{
${ }^{1}$ Assuming the intrinsic received signal width is $<\Delta b$, the spectral channel bandwidth
}

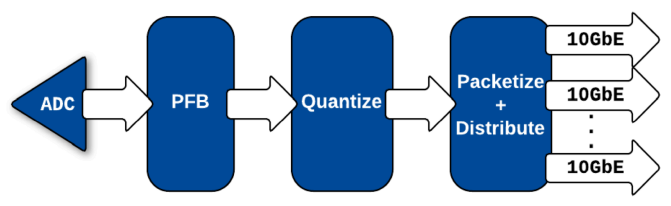

Figure 2: FPGA Design (symbolic). An analog stream is digitized, coarse channelized, quantized and transmitted over an Ethernet network. 


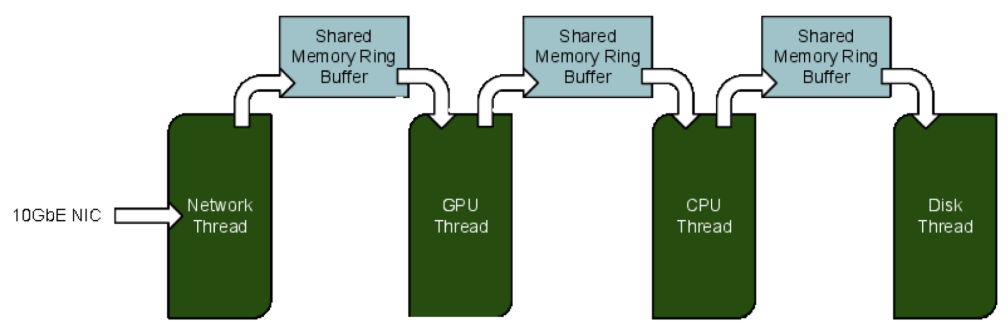

Figure 3: CPU/GPU software schematic diagram. Multiple software threads running on both CPU cores and graphics processing units (GPUs) interact through shared memory buffers. Data move from a network interface card (Network Thread) to a GPU for high resolution channelization (GPU thread) to the CPU for candidate identification (CPU Thread) and final candidates are written to disk (Disk Thread). Not shown are additional (not yet implemented) threads to perform interference excision and signal drift correction.

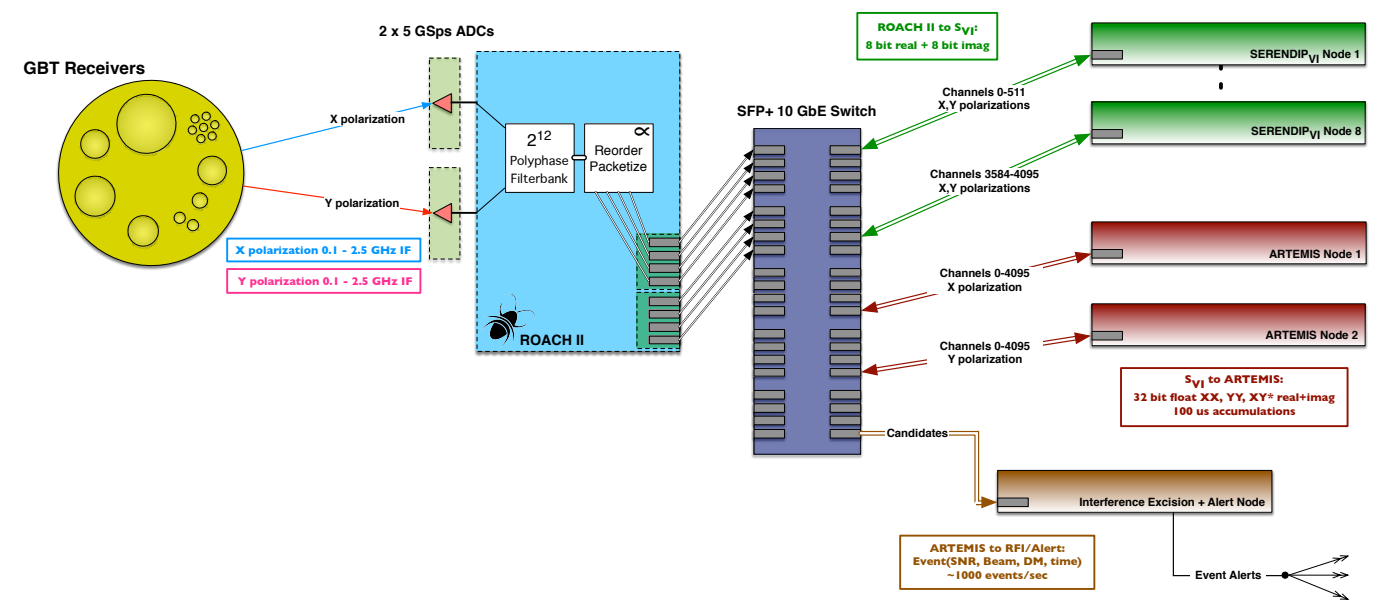

Figure 4: High level diagram of the Green Bank Telescope commensal digital backend. High-speed 5 GSps analog-to-digital converters (ADCs) and field-programmable gate array (FPGA) signal processing board sample an IF band between $0.1-2.5 \mathrm{GHz}$, coarsely channelize to $\sim 500 \mathrm{kHz}$ resolution and transmit complex voltages to commodity CPU/GPU servers via a $10 \mathrm{GbE}$ switch. Both SETI and fast radio burst search backends are shown attached to the system.

Acknowledgments The work described here is supported by grants from the National Aeronautics and Space Administration and the John Templeton Foundation.

\section{References}

[1] Giuseppe Cocconi and Philip Morrison. Searching for Interstellar Communications. Nature, 184(4):844-846, September 1959.

[2] F D Drake. Project OZMA. Physics Today, 14(4):40, 1961.

[3] P Horowitz, B S Matthews, J Forster, I Linscott, C C Teague, K Chen, and P Backus. Ultranarrowband searches for extraterrestrial intelligence with dedicated signal-processing hardware. Icarus (ISSN 0019-1035), 67:525-539, September 1986.

[4] D Leigh. An Interference-Resistant Search for Extraterrestrial Microwave Beacons. Harvard University PhD Thesis, 1998.

[5] E Korpela, D Werthimer, D Anderson, Jeff Cobb, and Matt Lebofsky. SETI@home - massively distributed computing for SETI. Computing in Science and Engineering, 3(1):78-83, January 2001.

[6] H Rampadarath, J S Morgan, S J Tingay, and C M Trott. The Very First Long Baseline Interferometric SETI Experiment. The Astronomical Journal, 144(2):38, June 2012.

[7] Andrew P. V. Siemion, Paul Demorest, Eric Korpela, Ron J Maddalena, Dan Werthimer, Jeff Cobb, Andrew W. Howard, Glen Langston, Matt Lebofsky, Geoffrey W Marcy, and Jill Tarter. A 1.1-1.9 GHz SETI Survey of the Kepler Field. I. A Search for Narrow-band Emission from Select Targets. The Astrophysical Journal, 767(1):94, April 2013.

[8] J Tarter. The search for extraterrestrial intelligence (SETI). Annual Reviews, 39:511-548, 2003.

[9] M Klein and S Gulkis. SETI-The microwave search problem and the NASA sky survey approach. The Search for Extraterrestrial Life: Recent ..., 1985.

[10] James M Cordes, T Joseph W Lazio, and Carl Sagan. Scintillation-induced Intermittency in SETI. Astrophysical Journal v.487, 487:782, October 1997.

[11] J Cordes. Astrophysical Masers as Amplifiers of ETI Signals. Third Decennial US-USSR Conference on SETI. ASP Conference Series, 47:257, 1993. 


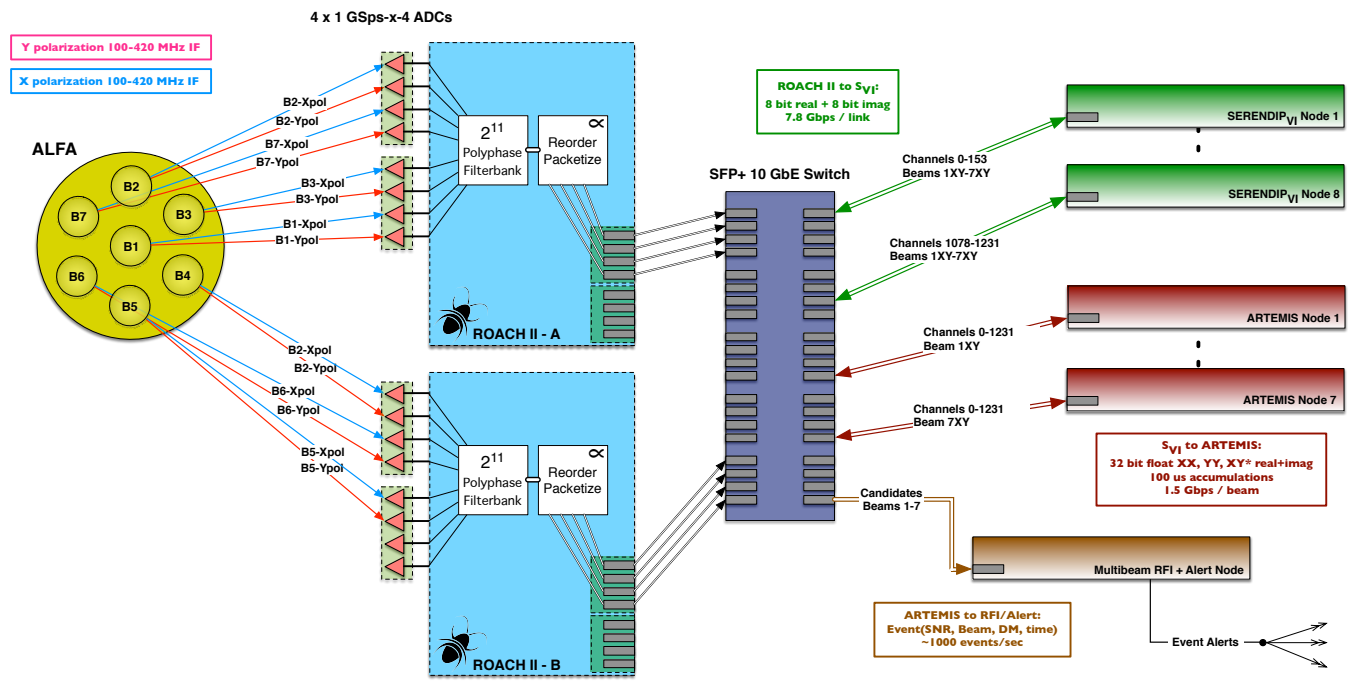

Figure 5: High level diagram of the Arecibo commensal digital backend. Analog signals from all seven beams of the ALFA receiver are digitized using high speed ADCs, fed through a 2048 point polyphase filterbank and distributed to SERENDIP VI compute nodes via an Ethernet switch. Each SERENDIP VI node detects and accumulates full Stokes spectra for a small portion of the band from all seven beams and passes these data along to ARTEMIS nodes where they are reformed into full-band spectra and searched for dispersed pulses. Ultimately, candidate pulse detections from all seven beams are analyzed together to identify dispersed pulses that are isolated on the celestial sphere; a key discriminant against terrestrial interference.

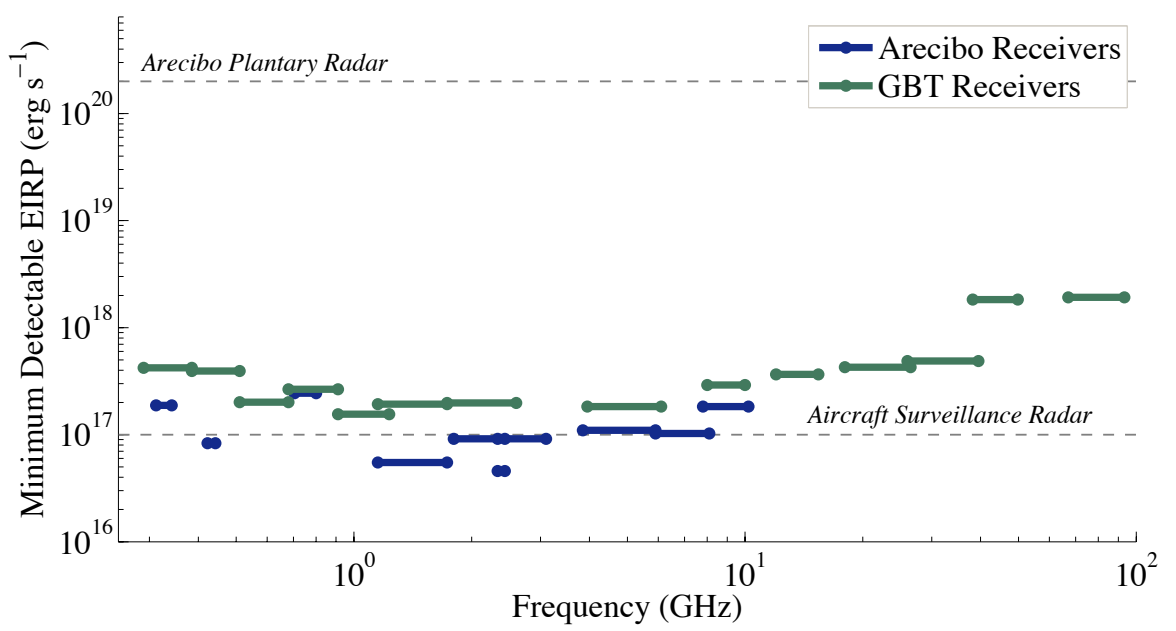

Figure 6: The sensitivity of observations conducted at Green Bank and Arecibo Observatory using the instrumentation described here, expressed as the minimum detectable equivalent isotropically radiated power (EIRP) using available heterodyne receivers on the GBT for a transmitter at 5 pc. For frequencies above $15 \mathrm{GHz}$ we assume a $50 \%$ weather quantile. See main text and technical justification for a discussion of other assumptions. For reference, a characteristic EIRP for high-power terrestrial surveillance radars is $\sim 10^{17} \mathrm{erg} \mathrm{s}^{-1}$, and the EIRP of the Arecibo Planetary Radar is $\sim 2 \times 10^{20} \mathrm{erg} \mathrm{sec}^{-1}$

[12] N L Cohen, M A Malkan, and JM Dickey. A passive SETI in globular clusters at the hydroxyl and water lines. Icarus, 41:198-204, 1980.

[13] D G Blair, R. P. Norris, E R Troup, R Twardy, K J Wellington, A J Williams, A E Wright, and M G Zadnik. A narrow-band search for extraterrestrial intelligence (SETI) using the interstellar contact channel hypothesis. Monthly Notices of the Royal Astronomical Society (ISSN 0035-8711), 257:105-109, July 1992.

[14] D Thornton, B Stappers, M Bailes, B R Barsdell, S D Bates, N D R Bhat, M Burgay, S Burke-Spolaor, D J Champion, P Coster, N D'Amico, A Jameson, S Johnston, M J Keith, M Kramer, L Levin, S Milia, C Ng, A Possenti, and W Van Straten. A Population of Fast Radio Bursts at Cosmological Distances. arXiv.org, (6):53-56, July 2013.

[15] S Bowyer, G Zeitlin, J Tarter, M Lampton, and W J Welch. The Berkeley parasitic SETI program. Icarus, 53:147, 1983.

[16] W A Gardner and C M Spooner. Signal interception: performance advantages of cyclic-feature detectors. IEEE Transactions on Communications, 40(1), 1992.

[17] W Armour, A Karastergiou, M Giles, C Williams, A Magro, K Zagkouris, S Roberts, S Salvini, F Dulwich, and B Mort. A GPU-based survey for millisecond radio transients using ARTEMIS. arXiv.org, November 2011.

[18] NRAO. GBT Proposer's Guide, November 2014. 